spiders collected. When a wasp has once :chosen a site for building, it is very difficult to drive her away.

63 , St. George Street, Leed;

Hy . LING ROTH

\section{The Microscope as a Refractor}

I AM rather surprised, after the judicious remarks of Dr. Gladstone on this subject in NATURE of July I (p. 192), to find Mr. Gordon Thompson still maintaining his opinion to have introduced anything not yet known or tried with the microscope adapted to this purpose. If he had had time to go over the papers of Royston Pigott (Proceedings of the Royal Society, 1876), of Mr. Sorby (Miniralogical Magazine, 1878), and of myself (Proceedings of the Royal Society, 1884), he could have convinced himself that all what he proposes has been already elaborated and applied. He could also have learnt why the method with the microscope is limited in its exactitude to the third decimal, as the mathematical expression which it involves is deduced from not very strict principles, this being as well the case with the formula for the hollow prism.

The Hague, July 2 I

L. BLEEKRODE

\title{
HERRMANN ABICH
}

$A^{S}$ briefly reported in NATURE last week this venerable geologist died at Vienna on July I. As far back as the year I 83 I he began his scientific career by the publication of an important memoir, in which by novel methods of chemical analysis he determined the composition of various minerals of the Spinel family, and showed how alike by chemical composition and crystalline form they could all be ranged in one group. This early paper gave evidence of the carefulness of observation which distinguished him through life. It was followed by other chemical and mineralogical essays, especially in the department of volcanic products. Gradually he was led to devote special attention to the phenomena of volcanic action, and in the course of his investigations to visit most of the volcanic districts of Europe. His folio atlas of views illustrative of Vesuvius and Etna (1837), and his "Vulkanische Bildungen" (184I), are among the best known of his writings. $\mathrm{He}$ had great facility as a sketcher, and some of his drawings of volcanic craters have done duty for nearly half a century in text-books in many languages. The east of Europe presented a wide and almost unknown field for his exploration. As far back as 1840 he published notices of his wanderings in the Caucasus. He ascended to the summit of Mount Ararat, and devoted most of the remainder of his life to the investigation of the vast region of the Caucasus and south-eastern Europe. Many papers published from time to time in the scientific journals record his unwearied industry. But perhaps the most striking and durable monument of his scientific achievements is his great work, "Geologische Forschungen in den Kaukasischen Ländern," the publication of which he was superintending at the time of his death. This magnificent monograph, of which only the first part has been published, brings before the reader in a series of maps, sketches, large panoramic views, and detailed descriptions a picture of the external aspect and geological structure of the Caucasian region and impresses him with a profound admiration for the author's geological prowess. Abich had during the last few years settled in Vienna, availing himself of the typographic facilities to be found in the Austrian capital. He has been a notable instance of the longevity attained by many active fiel $\downarrow$-geologists, for he almost reached the age of three score and ten years, retaining to the end his enthusiasm and industry. It is to be hoped that the second part of his monumental work, which is to treat of the eastern half of the Armenian Highlands, has been left in such a state as to admit of publication.

\section{CAPILLARY ATTRACTION ${ }^{1}$}

II.

Y OW in this second way we have, in performing the folding motion, allowed the water surface to become less by 60 square centimetres. It is easily seen that, provided the radius of curvature in every part of the surface exceeds one or two hundred times the extent of distance to which the molecular attraction is sensible, or, as we may say practically, provided the radius of curvature is everwhere greater than 5000 micro-millimetres (that is, the two-hundredth of a millimetre), we should have obtained this amount of work with the same diminution of water-surface, however performed. Hence our result is that we have found $45 / 60$ (or $3 / 40$ ) of a centimetre-gramme of work per square centimetre of diminution of surface. This is precisely the result we should have had if the water had been absolutely deprived of the attractive force between water and water, and its whole surface had been coated over with an infinitely thin contractile film possessing a uniform contractile force of $3 / 40$ of a gramme weight, or 75 milligrammes, per lineal centimetre.

It is now convenient to keep to our ideal film, and give

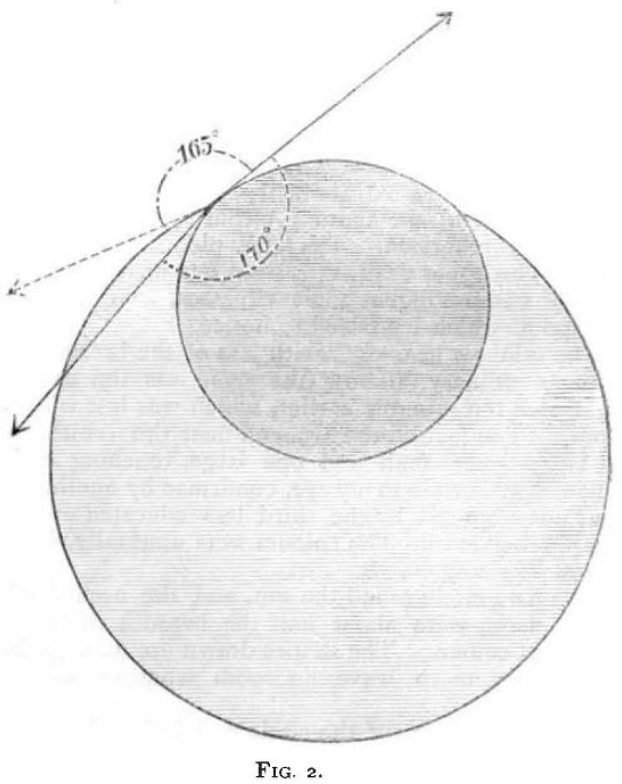

up thinking of what, according to our present capacity for imagining molecular action, is the more real thingnamely, the mutual attraction between the different portions of the liquid. But do not, I entreat you, fall into the paradoxical habit of thinking of the surface film as other than an ideal way of stating the resultant effect of mutual attraction between the different portions of the fluid. Look, now, at one of the pieces of water ideally rigidified, or, if you please, at the two pieces put together to make one. Remember we are at the centre of the earth. What will take place if this piece of matter resting in the air before you suddenly ceases to be rigid? Imagine it, as I have said, to be enclosed in a film everywhere tending to contract with a force equal to $3 / 40$ of a gramme or 75 milligrammes weight per lineal centimetre. This contractile film will clearly press most where the convexity is greatest. A very elementary piece of mathematics tells us that on the rigid convex surface which you see, the amount of its pressure per square centimetre will be found by multiplying the sum ${ }^{2}$ of the curvatures in two mutually-perpendicular normal sections

$x$ Continued from p. 272

2 This sum for brevity I henceforth call simply "the curvature of the surface" at any point. 
by the amount of the force per lineal centimetre. In any place where the surface is concave the effect of the surface tension is to suck outwards--that is to say, in mathematical language, to exert negative pressure inwards. Now, suppose in an instant the rigidity to be annulled, and the piece of glass which you see, still undisturbed by gravity, to become water. The instantaneous effect of these unequal pressures over its surface will be to set it in motion. If it were a perfect fluid it would go on vibrating for ever with wildly-irregular vibrations, starting from so rude an initial shape as this which I hold in my hand. Water, as any other liquid, is in reality viscous, and therefore the vibrations will gradually subside, and the piece of matter will come to rest in a spherical figure, slightly warmed as the result of the work done by the forces of mutual attraction by which it was set in motion from the initial shape. The work done by these forces during the change of the body from any one shape to any other is in simple proportion to the diminution of the whole surface area ; and the configuration of equilibrium, when there is no disturbance from gravity, or from any other solid or liquid body, is the figure in which the surface area is the smallest possible that can enclose the given bulk of matter.

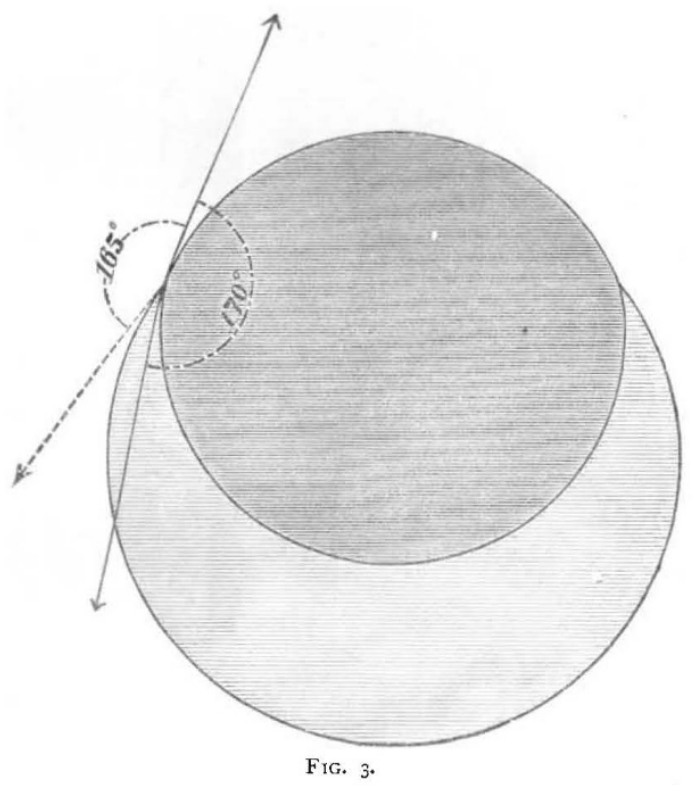

I have calculated the period of vibration of a sphere of water ${ }^{1}$ (a dew-drop !) and find it to be $\frac{1}{4} a^{\frac{3}{2}}$, where $a$ is the radius measured in centimetres; thus-

For a radius of $\frac{1}{4} \mathrm{~cm}$. the period is $\frac{1}{3} \frac{3}{1}$ second

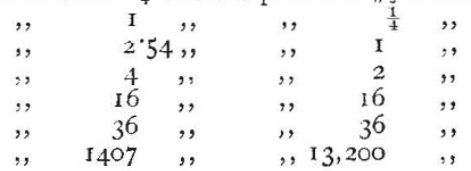

The dynamics of the subject, so far as a single liquid is concerned, is absolutely comprised in the mathematics without symbols which I have put before you. 'Twenty pages covered with sextuple integrals could tell us no more.

Hitherto we have only considered mutual attraction between the parts of two portions of one and the same liquid-water for instance. Consider, now, two different kinds of liquid: for instance, water and carbon disulphide (which, for brevity, I shall call sulphide). Deal with them

\footnotetext{
${ }^{1}$ See paper by Lord Rayleigh in Proc. Koy. Soc., No. 195, May 5, 1879 .
}

exactly as we dealt with the two pieces of water. I need not go through the whole process again; the result is obvious. Thirty times the excess of the sum of the surfacetensions of the two liquids separately, above the tension of the interface between them, is equal to the work done in letting the two bodies come together directly over the

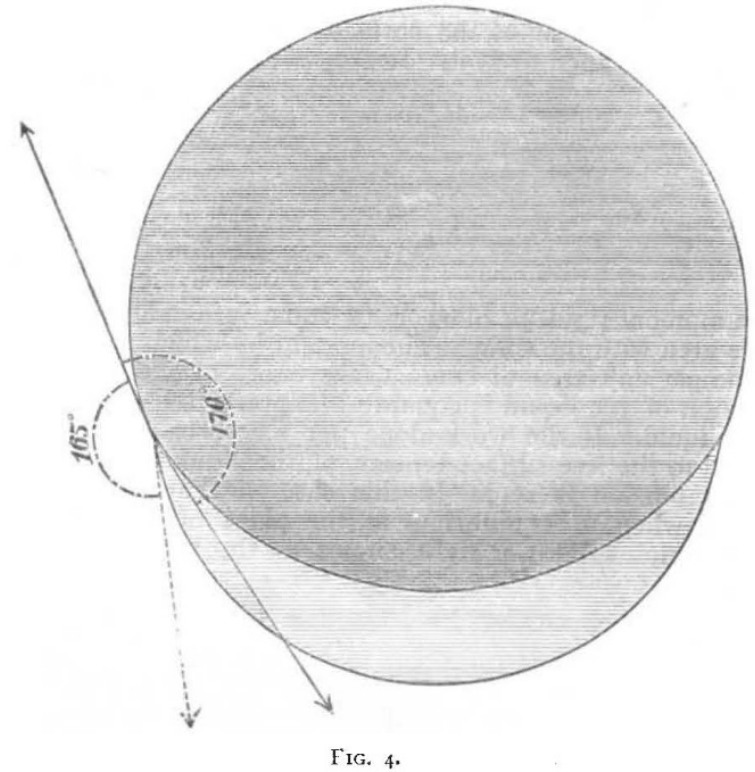

supposed area of thirty square centimetres. Hence the interfacial tension per unit area of the interface is equal to the excess of the sum of the surface-tensions of the trwo liquids separately, above the work done in letting the two bodies come together directly so as to meet in a unit area of each. In the prticular case of two similar bodies

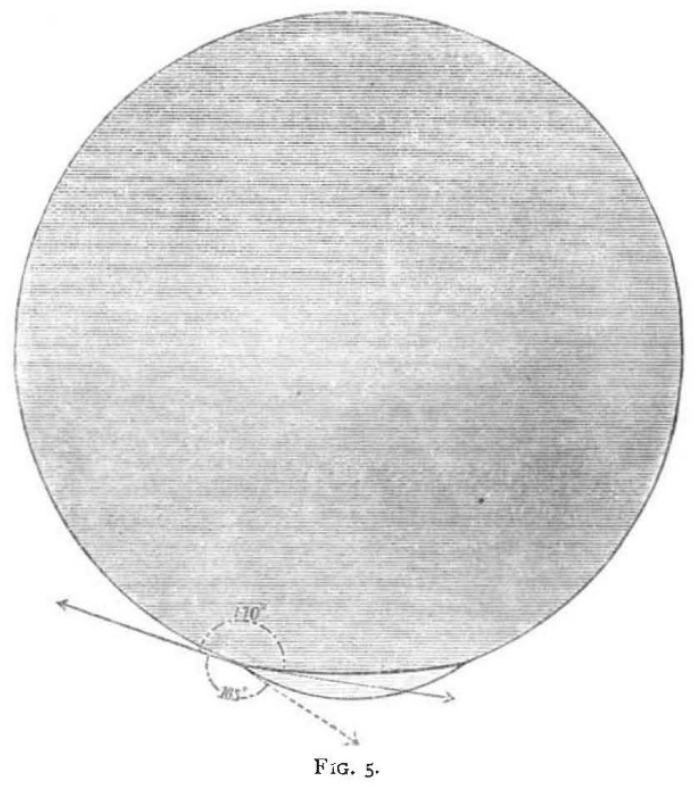

coming together into ferfect contact, the interfacial tension must be zero, and therefore the work done in letting them come together over a unit area must be exactly equal to twice the surface-tension; which is the case we first considered.

If the work done between two different liquids in letting 
them come together over a small area, exceeds the sum of the surface-tensions, the interfacial tension is negative. The result is an instantaneous puckering of the interface, as the commencement of diffusion and the well-known process of continued inter-diffusion follows.

Consider next the mutual attraction between a solid and a liquid. Choose any particular area of the solid, and let a portion of the surface of the liquid be preliminarily shaped to fit it. Let now the liquid, kept for

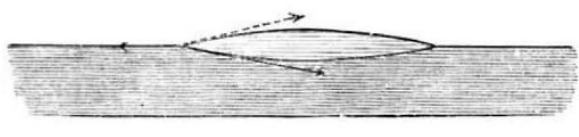

FIG. 6.

the moment rigid, be allowed to come into contact over this area with the solid. The amount by which the work done per unit area of contact falls short of the surfacetension of the liquid is equal to the interfacial tension of the liquid. If the work done per unit area is exactly equal to the free-surface tension of the liquid, the interfacial tension is zero. In this case the surface of the liquid when in equilibrium at the place of meeting of liquid and solid is at right angles to the surface of the

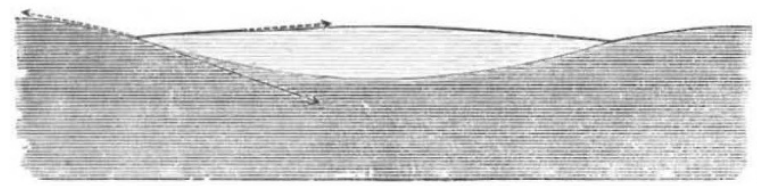

Fi3. 7.

solid. The angle between the free surfaces of liquid and solid is acute or obtuse according as the interfacial tension is positive or negative ; its cosine being equal to the interfacial tension divided by the free-surface tension. The greatest possible value the interfacial tension can have is clearly the free-surface tension, and it reacbes this limiting value only in the, not purely static, case of a liquid resting on a solid of high thermal conductivity, kept at a temperature greatly above the boiling-point of

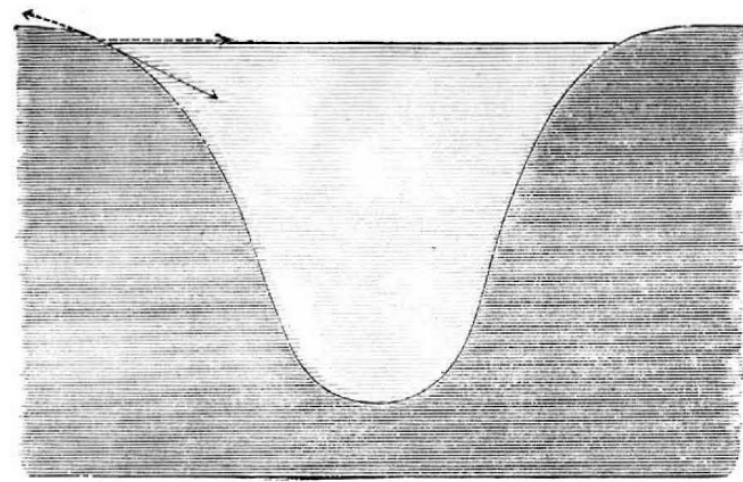

FIG. 8.

the liquid; as in the well-known phenomena to which attention has been called by Leidenfrost and Boutigny. There is no such limit to the absolute value of the interfacial tension when negative, but its absolute value must be less than that of the free surface tension to admit of equilibrium at a line of separation between liquid and solid. If minus the interfacial tension is exactly equal to the free-surface tension, the angle between the free surfaces at the line of separation is exactly $180^{\circ}$. If minus the interfacial tension exceeds the free-surface tension, the liquid runs all over the solid, as, for instance, water over a glass plate which has been very perfectly cleansed. If for a moment we leave the centre of the earth, and suppose ourselves anywhere else in or on the earth, we find the liquid running up, against gravity, in a thin film over the upper part of the containing vessel, and leaving the interface at an angle of $180^{\circ}$ between the free surface of the liquid, and the surface of the film adhering to the solid above the bounding line of the free liquid surface. This is the case of water contained in a glass vessel, or in contact with a piece of glass of any shape, provided the surface of the glass be very perfectly cleansed.

When two liquids which do not mingle, that is to say, two liquids of which the interfacial tension is positive, are placed in contact and left to themselves undisturbed by gravity (in our favourite laboratory in the centre of the earth suppose), after performing vibrations subsiding in virtue of viscosity, the compound mass will come to rest, in a configuration consisting of two in-

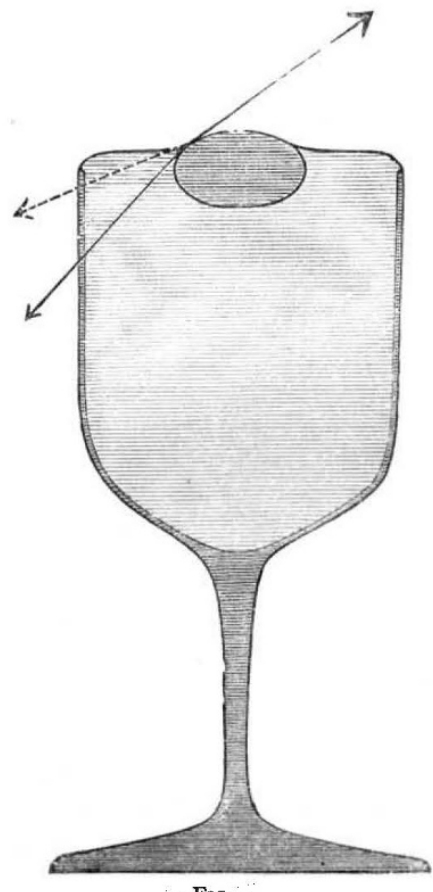

Fig. 9.

tersecting segments of spherical surfaces constituting the outer boundary of the two portions of liquid, and a third segment of spherical surface through their intersection constituting the interface between the two liquids. These three spherical surfaces meet at the same angles as three balancing forces in a plane whose magnitudes are respectively the surface tensions of the outer surfaces of the two liquids and the tension of their interface. Figs. 2 to 5 illustrate these configurations in the case of bisulphide of carbon and water for several different proportions of the volumes of the two liquids. (In the figures the dark shading represents water in each case.) When the volume of each liquid is given, and the angles of meeting of the three surfaces are known, the problem of describing the three spherical surfaces is clearly determinate. It is an interesting enough geometrical problem.

If we now for a moment leave our gravitationless laboratory, and, returning to the Theatre of the Royal Institution, bring our two masses of liquid into contact, as I now do in this glass bottle, we have the one liquid floating upon the other, and the form assumed by the floating liquid may be learned, for several different cases, from the phenomena exhibited in these bottles 
and glass beakers, and shown on an enlarged scale in these two diagrams (Figs. 6 to 8 ); which represent bisulphide of carbon floating on the surface of sulphate of zinc, and in this case (Fig. 8) the bisulphide of carbon drop is of nearly the maximum size capable of floating. Here is the bottle whose contents are represented in Fig. 8 , and we shall find that a very slight vertical disturbance serves to submerge the mass of bisulphide of carbon. There now it has sunk, and we shall find when its vibrations have ceased that the bisulphide of carbon has taken the form of a large sphere supported within the sulphate of zinc. Now, remembering that we are again at the centre of the earth, and that gravity does not hinder us, suppose the glass matter of the bottle

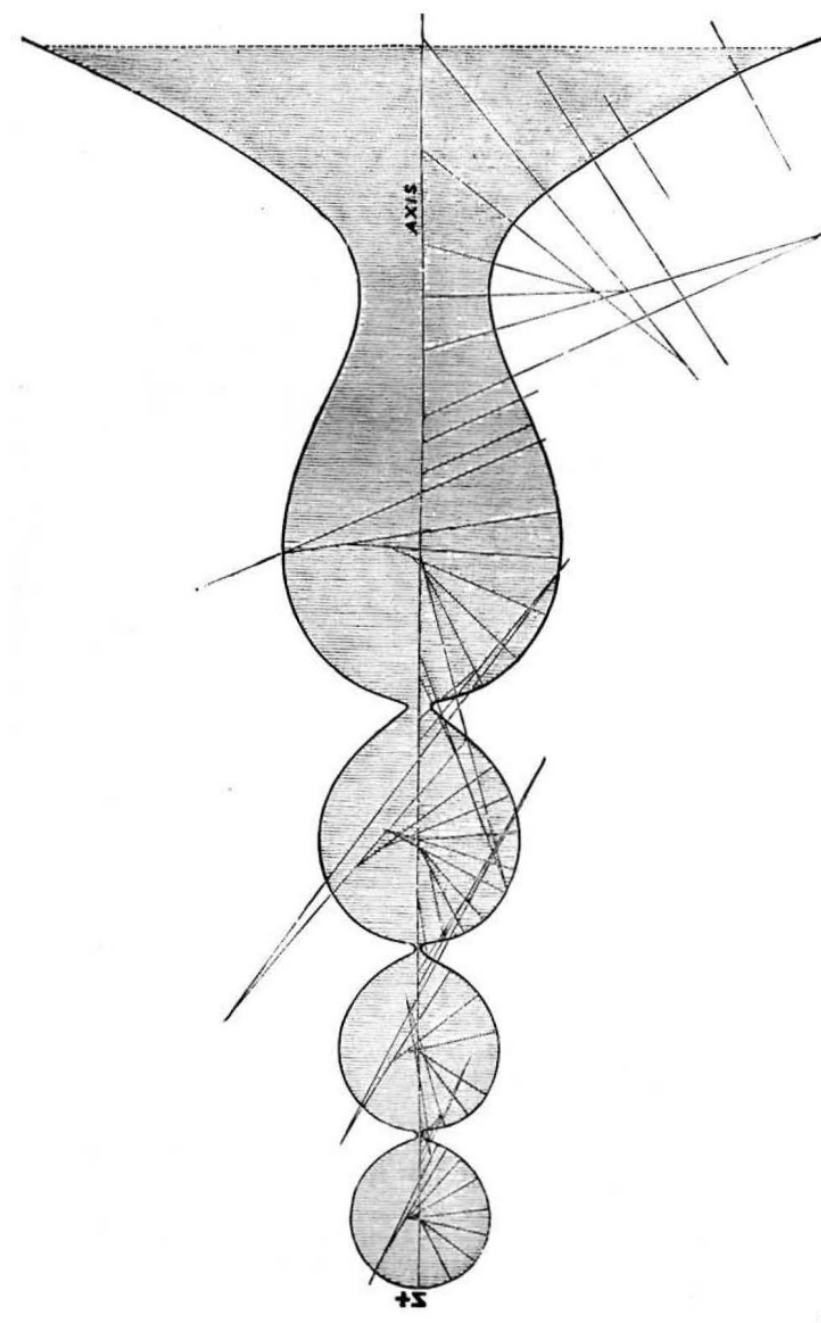

FI $;, 10$.

suddenly to become liquid sulphate of zinc, this mass would become a compound sphere like the one shown on this diagram (Fig. 3), and would have a radius of about 8 centimetres. If it were sulphate of zinc alone, and of this magnitude, its period of vibration would be about $5 \frac{1}{2}$ seconds.

Fig. 9 shows a drop of sulphate of zinc floating on a wine-glassful of bisulphide of carbon.

In observing the phenomena of two liquids in contact, I have found it very convenient to use sulphate of zinc (which I find, by experiment, has the same free-surface tension as water) and bisulphide of carbon; as these liquids do not mix when brought together, and, for a short time at least, there is no chemical interaction between them. Also, sulphate of zinc may be made to have a density less than, or equal to, or greater than, that of the bisulphide, and the bisulphide may be coloured to a more or less deep purple tint by iodine, and this enables us easily to observe drops of any one of these liquids on the other. In the three bottles now before you the clear liquid is sulphate of zinc-in one bottle it has a density less than, in another equal to, and in the third greater than, the density of the sulphide - and you see how, by means of the coloured sulphide, all the phenomena of drops resting upon or floating within a liquid into which they do not diffuse may be observed, and, under suitable arrangements, quantitatively estimated.

When a liquid under the influence of gravity is supported by a solid, it takes a configuration in which the difference of curvature of the free surface at different levels is equal to the difference of levels divided by the surface tension reckoned in terms of weight of unit bulk of the liquid as unity ; and the free surface of the liquid leaves the free surface of the solid at the angle whose

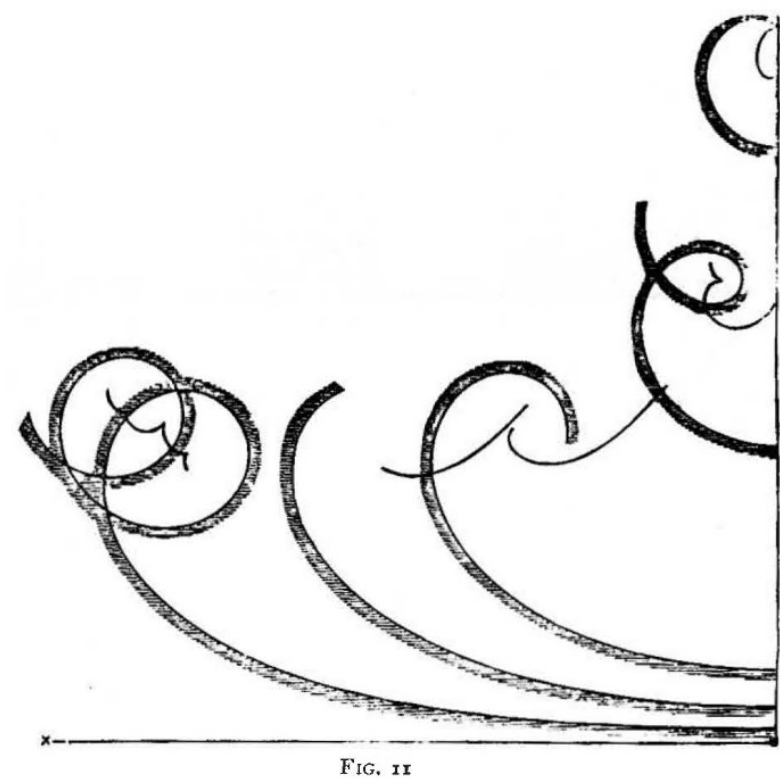

cosine is, as stated above, equal to the interfacial tension divided by the free-surface tension, or at an angle of $180^{\circ}$ in any case in which minus the interfacial tension exceeds the free-surface tension. The surface equation of equilibrium and the boundary conditions thus stated in words, suffice fully to determine the configuration when the volume of the liquid and the shape and dimensions of the solid are given. When I say determine, I do not mean unambiguously. There may of course be a multinlicity of solutions of the problem; as, for instance, when the solid presents several hollows in which, or projections hanging from which, portions of the liquid, or in or hanging from any one of which the whole liquid, may rest.

When the solid is symmetrical round a vertical axis, the figure assumed by the liquid is that of a figure of revolution, and its form is determined by the equation given above in words. A general solution of this problem by the methods of the differential and integral calculus transcends the powers of mathematical analysis, but the following simple graphical method of working out what constitutes mathematically a complete solution, occurred to me a great many years ago.

Draw a line to represent the axis of the surface of revo- 
lution. This line is vertical in the realisation now to be given, and it or any line parallel to it will be called vertical in the drawing, and any line perpendicular to it will be called horizontal. The distance between any two horizontal lines in the drawing will be called difference of levels.

Through any point, $\mathrm{N}$, of the axis draw a line, $\mathrm{N} \mathrm{P}$, cutting it at any angle. With any point, $O$, as centre on the line $\mathrm{N} \mathrm{P}$, describe a very small circular arc through $\mathrm{P} \mathrm{P}^{\prime}$, and let $\mathrm{N}^{\prime}$ be the point in which the line of $O \mathrm{P}^{\prime}$ cuts the axis. Measure N P, $\mathrm{N}^{\prime} \mathrm{P}^{\prime}$, and the difference of levels between $\mathrm{P}$ and $\mathrm{P}^{\prime}$. Denoting this last by $\delta$, and taking $a$ as a linear parameter, calculate the value of

$$
\left(\frac{\delta}{a^{2}}+\frac{\mathrm{I}}{\mathrm{OP}}+\frac{\mathrm{I}}{\mathrm{NP}}-\frac{\mathrm{I}}{\mathrm{N}^{\prime} \mathrm{P}^{\prime}}\right)^{-1} \text {. }
$$

Take this length on the compasses, and putting the pencil point at $\mathrm{P}^{\prime}$, place the other point at $\mathrm{O}^{\prime}$ on the line $\mathrm{P}^{\prime} \mathrm{N}^{\prime}$, and with $\mathrm{O}^{\prime}$ as centre, describe a small arc, $\mathrm{P}^{\prime} \mathrm{P}^{\prime \prime}$. Continue the process according to the same rule, and the successive very small arcs so drawn will constitute a curved line, which is the generating line of the surface of revolution inclosing the liquid, according to the conditions of the special case treated.

This method of solving the capillary equation for surfaces of revolution remained unused for fifteen or twenty years, until in $1874 \mathrm{I}$ placed it in the hands of Mr. John Perry (now Professor of Mechanics at the City and Guilds Institute), who was then attending the Natural Philosophy Laboratory of Glasgow University. He worked out the problem with great perseverance and ability, and the result of his labours was a series of skilfully executed drawings representing a large variety of cases of the capillary surfaces of revolution. These drawings, which are most instructive and valuable, I have not yet been able to prepare for publication, but the most characteristic of them have been reproduced on an enlarged scale, and are now on the screen before you. ${ }^{7}$ Three of the diagrams, those to which I am now pointing (Figs. IO, II, and I2), illustrate strictly theoretical solutions-that is to say, the curves there shown do not represent real capillary sur-

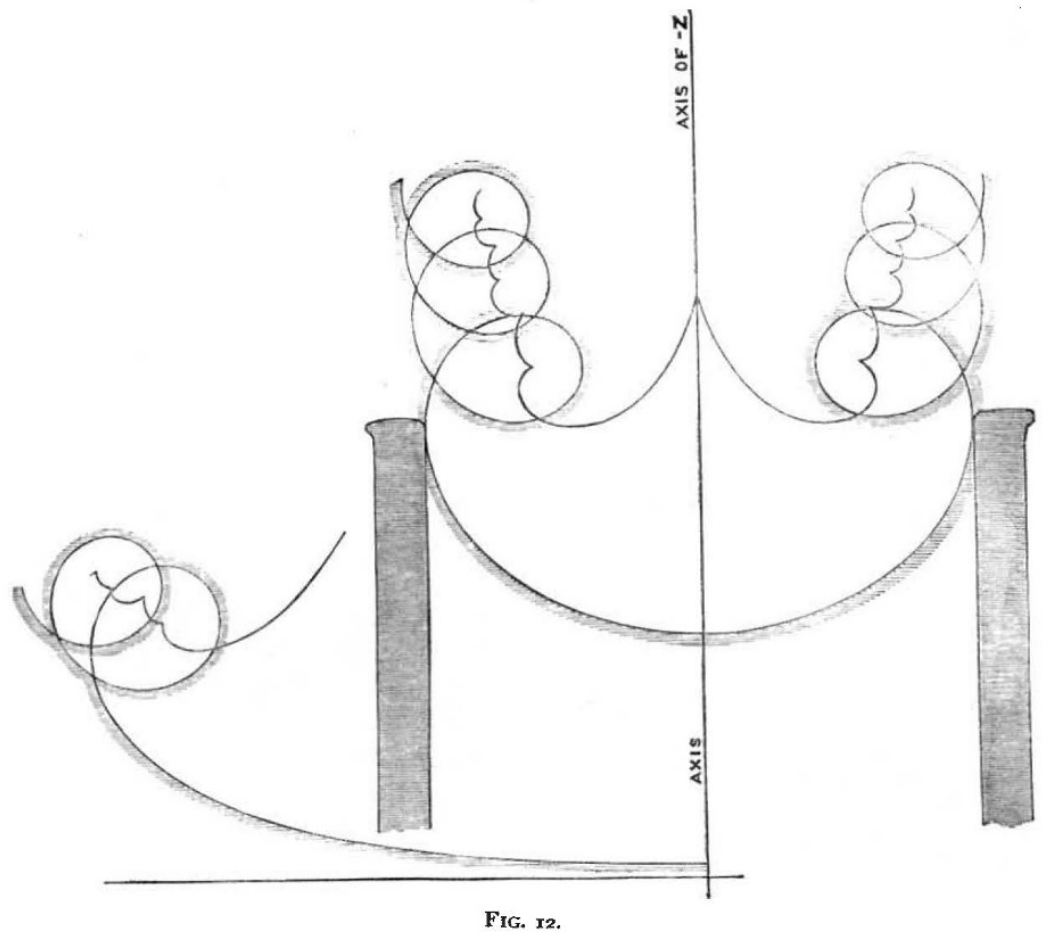

faces-but these mathematical extensions of the problem, harmony with the views of the best friends of scientific while most interesting and instructive, are such as cannot education in this country.

be adequately treated in the time now at my disposal.

WILLIAM THOMSON

(To be continued.)

Solution.-Disappearance of a solid in a liquid by solution. Saturation of a liquid. Effect of increase of temperature on saturation. Effect of lowering the temperature on saturation. Crystallisation. Filtration. Solvent properties of water. Rain, spring, river, and pond waters, \&c. Solid matter in different waters; how estimated. Loch Katrine water. Thames water.

THE SCIENCE AND ART DEPARTMENT EXAMINATION IN CHEMISTRY

THE new editor of the "Science and Art Directory" announces a new departure of the most important kind in the teaching of chemistry. In addition to the oral instruction in the elementary stage, there is now introduced an alternative first stage or elementary course intended for those students who only require the elements of chemistry as a foundation for their studies in other subjects.

We give the new syllabus so that it may speak for itself, and congratulate the Department on a step in Sea water. Hard and soft waters. Mineral waters. Similar solvent of other liquids. Solution of one liquid in another. Liquids insoluble in one another. Solution of gases in water and other liquids. The effect of heat on the quantity of gas dissolved by a Jiquid.

Experiments. - Suspend a piece of white sugar by a thread in a glass vessel containing water. Dissolve salt in water. Show on a balance that sugar or salt and water when separate and when dissolved weigh the same. Show that salt is obtained t The diagrams here referred to are now published in Figs. ro to 24 of the present report of the lecture at the Royal Institution. These figures are accurate copies of Mr. Perry's original drawings, and I desire to acknowledge the great care and attention which Mr. Cooper, engraver to NATURE,
has given to the work. 